\title{
Risk communication in the context of clinical research
}

Paulo NADANOVSKY(a)

Luciane Rezende COSTA ${ }^{(b)}$

Ana Paula Pires dos SANTOS(c)

(a)Fundação Oswaldo Cruz - Fiocruz, Escola Nacional de Saúde Pública, Departamento de Epidemiologia e Métodos Quantitativos em Saúde, Rio de Janeiro-RJ, Brasil.

(b) Universidade Federal de Goiás, Faculdade de Odontologia, Goiânia, GO, Brasil.

(c) Universidade do Estado do Rio de Janeiro - UERJ, Faculdade de Odontologia, Departamento de Odontologia Preventiva e Comunitária, Rio de Janeiro, RJ, Brasil.

Declaration of Interests: The authors certify that they have no commercial or associative interest that represents a conflict of interest in connection with the manuscript.

\section{Corresponding Author:}

Paulo Nadanovsky

E-mail:nadanovsky@ensp.fiocruz.br
Abstract: Physicians and dentists usually make clinical decisions and recommendations without a clear understanding of the meaning of the numbers regarding the accuracy of diagnostic tests and the efficacy of treatments. This critical review aimed to identify problems in the communication of diagnostic test accuracy and treatment benefits and to suggest strategies to improve risk communication in these contexts. Most clinical decisions are taken under uncertainty. Health professionals cannot predict the outcome in one individual patient. This uncertainty invites these professionals to make decisions based on heuristics, which gives rise to several cognitive biases. Cognitive biases are automatic and unconscious, so how is it possible to mitigate their undesirable effects on risk interpretation in the context of clinical practice? Some forms of risk communication reinforce cognitive bias, while others weaken them. Maybe one of the most difficult obstacles to overcome is the difficulty to think with numbers. This difficulty probably arises from a mismatch of ancestral adaptations of the brain having to deal with modern environments, which are quite different from the ancestral ones. There are two quite common, but bad, forms of risk communication: the conditional probability and the relative risk reduction or efficacy. People, including physicians and dentists, are confused with this kind of information. The main methods discovered so far to facilitate a clearer understanding are to emphasize the base rates of the events and to use absolute numbers, that is to use natural frequencies, instead of percentages and conditional probabilities.

Keywords: Risk Assessment; Risk Management; Evidence-Based Practice.

\section{Introduction}

The interpretation of evidence to inform health care decisions depends on the transparent communication of risk, among other factors. This critical review aimed to identify problems in the communication of diagnostic test accuracy and treatment benefits and to suggest strategies to improve risk communication in these contexts.

\section{Medical convictions can be delusional: the PSA and the preventive colonoscopy}

Some years ago, between my 40s and 50s (PN), my doctor told me I had reached an age in which I should begin to undergo periodical exams for 
prostate cancer detection, the PSA (Prostate-Specific Antigen). He did not give me any information about the risk of a man of my age developing prostate cancer or dying from prostate cancer. He also did not tell me the extent to which these periodical exams would reduce my risk of dying from prostate cancer. Fortunately, by that time, I had been teaching evidence-based clinical practice for many years, and prostate cancer was one of the examples I used in my classes. Therefore, I was aware of two things: a) The risk of a 50-60-year-old carioca dying in the next 10 years was 135 in 1,000; from prostate cancer, only 1 in 1,000; that is my probability of dying from prostate cancer was relatively very small. b) The periodical PSA assessment does not reduce the risk of dying from prostate cancer, and worse than this, it increases my risk of unnecessary surgery and its frequent negative consequences, such as urinary incontinence and sexual impotence. Let us see the numbers. For every 1,000 symptomless $45-80$-yearold men, without the PSA, 68 will be diagnosed with prostate cancer, seven will die from prostate cancer, and 61 will be treated and survive cancer. With PSA, 88 will be diagnosed with prostate cancer, seven will die from prostate cancer, and 81 will be treated and survive cancer. Thus, due to PSA screening, no lives will be saved, and approximately 20 men will be diagnosed and treated for cancer that would not have caused them any harm. ${ }^{1,2}$

My doctor stopped insisting on the PSA but then started recommending colonoscopy screening for colorectal cancer. Dear reader, I like and trust my doctor very much. He is a very competent doctor and a genuinely nice person. The point here is not to say that there is a problem with him. We argue that there is a real and widespread problem that encompasses all health professionals and beyond the health field. There is a problem of communicating risks, which makes it difficult for society as a whole and each person individually to make rational decisions. We are trying to show how this problem occurs in medicine, dentistry, and health sciences in general.

So, back to the colonoscopy. This example was drawn from an article published in the New England Journal of Medicine (NEJM), in which the editor selected "a dozen of articles that changed practice since 2000". ${ }^{3}$ Let us turn to the numbers again. The mortality from colorectal cancer in the 15 years of follow-up was compared between patients who had adenomas removed (colonoscopy) and the general population. ${ }^{4}$ There was a $53 \%$ mortality reduction in the colonoscopy group in comparison to the general population! ${ }^{4}$ Probably this was the result that encouraged my $(\mathrm{PN})$ doctor and others to recommend colonoscopy screening, and that made the NEJM chief editor select this article as one of the most influential since $2000 .{ }^{3}$ However, $53 \%$ efficacy or relative risk reduction does not mean that $53 \%$ of the patients benefit from colonoscopy! Many people believe that $53 \%$ efficacy means that, for every 100 people who undergo a colonoscopy and have adenomas removed, 53 have their lives saved. However, this is an illusion very distant from reality. To understand what this 53\% efficacy means, let us improve risk communication. This 53\% efficacy derived from the following numbers: 2,602 patients underwent colonoscopic removal of adenomatous polyps, and 12 died from colorectal cancer in the 15 years of follow-up, whereas in the general American population, there were 25.4 deaths from colorectal cancer. This difference of 13.4 deaths, that is 25.4 minus 12 , means a $53 \%$ reduction - 13.4 correspond to $53 \%$ of 25.4 . To improve the risk communication even more, these numbers can be transformed into rates per 1,000 for the 15 years of follow-up: there were approximately 5 deaths from colorectal cancer in the colonoscopy group and 10 in the general American population. Thus, colonoscopy reduced the risk from 10 in 1,000 to 5 in 1,000 . Note that this is a $0.5 \%$ and not $53 \%$ absolute benefit.

Besides the absolute numbers being much more modest than the $53 \%$ efficacy implies, it is important to note that this study that changed medical practice was not randomized - it did not compare a group randomly allocated to colonoscopy to a group randomly allocated to no colonoscopy. ${ }^{4}$ Thus, it is likely that the groups of this study (colonoscopy versus general population) were not comparable. The mortality rate from all causes was higher in the general population than in the colonoscopy group, which might have distorted the results. Generally, screened groups have a healthier 
lifestyle and a better socioeconomic condition, which reduces the risk of death irrespective of screening. Therefore, the approximate reduction from 10 to 5 deaths in 1,000 people attributed to the colonoscopy may be overestimated in this study. Additionally, the risk of dying from any cause in the group of colonoscopy was 479 in 1,000 in the 15 years of follow-up, which means that the risk of dying from colorectal cancer is relatively small in comparison to any other cause.

To sum up, there might be a mortality reduction due to periodical colonoscopy screening. Still, it appears to be modest in absolute terms and uncertain given the methodological limitations of the best available scientific evidence. Thus, until results from randomized controlled clinical trials become available, physicians should be less assertive and more informative when recommending screening colonoscopy to their patients.

We will see that physicians and dentists get confused with these percentage reductions, such as the $53 \%$ presented in the colonoscopy study. They usually make decisions and recommendations without a clear understanding of what these numbers mean.

\section{Human illusions}

This overconfidence, common among physicians and illustrated in the PSA and colonoscopy examples, has deep roots in human nature. Among the many challenges to solve this problem, there is the illusion of certainty - an innate attribute, common to all human beings. We have illusions about certainties, even when reality shows our certainties are not based on facts. We tend to overestimate our opinions as if they were unique and proven truths.

Physicians and dentists experience these illusions in their professional activities. For instance, they usually have the illusion that treatment have only benefits and no harm; that there is one and only one better treatment; that a diagnostic test is absolutely correct. This illusion of certainty in health practice is a mental barrier that hinders a well informed and data-based decision-making process. ${ }^{5}$

Daniel Kahneman, a psychologist winner of the Nobel Prize in Economics, made the alert: "An unbiased appreciation of uncertainty is a cornerstone of rationality - but it is not what organizations want. Extreme uncertainty is paralyzing under dangerous circumstances, and the admission that one is merely guessing is especially unacceptable when the stakes are high. Acting on pretended knowledge is often the preferred approach". ${ }^{6}$

\section{Illusory risks about breast cancer demonstrated by Gerd Gigerenzer}

The illusion of certainty is spread to the public, who adopts a distorted view of reality. For instance, there is a disparity between the actual risk of dying from breast cancer and the risk believed by women. Additionally, there is a great disparity between the actual protective effect of mammography screening and what women believe to be the protective effect of this type of screening. It is common to find leaflets in campaigns saying that one in ten women develops breast cancer. This information, "one in ten," has become a mantra in the popular media and breast cancer screening programs. This number has terrified many women, but what does it mean? What is not explained is that this number refers to a woman's cumulative chances of developing breast cancer by the age of 85 . However, most women die before this age, and those who develop cancer in this advanced age will probably die from another cause. ${ }^{5}$ Let us look at the numbers.

To make it easier, we shall look at the absolute numbers of events for every 1,000 people. The scenario is a group of 1,000 women followed since birth until 84 years of age (data from Ontario, Canada Brazilian women have lower breast cancer risk than Canadians). For instance, four women developed breast cancer in their 30s and 13 in their 40s; at 84 , the total number of breast cancer cases added 99 cases, which corresponds to the numbers "one in ten" (that is almost 100 in 1,000). Thirty-three of the women died from breast cancer. The numbers "one in ten" gives the impression that, at any age, the risk of a woman developing breast cancer is one in ten, maybe in the next five or ten years. As we have seen, this is not true; this is the cumulative risk of an entire life, at the age of 84 . Besides, it may also give the impression that the risk of dying from breast cancer is one in ten, but it is actually much 
lower than one in ten (it was 33 in 1,000 from birth to the age of 84 in this group of Canadian women). Also, the risk of dying from other causes is much higher than from breast cancer. In contrast to 33 deaths from breast cancer, there were 203 from cardiovascular causes, and 330 from other causes since birth until age $84 .{ }^{5}$

How do women interpret these numbers? In a group of well-educated American women aged 40-49, with no history of breast cancer, each of them was asked to imagine 1,000 women exactly like herself and to estimate how many of these women would die from breast cancer in the next 10 years. On average, they estimated that this risk would be of 100 in 1,000, that is exactly "one in ten". This was an overestimate of 20 times the actual risk of dying from breast cancer. Among 1,000 45-year-old women (the mean age in this study), five died from breast cancer, not 100, in the following ten years. ${ }^{5}$ We asked the same question in Brazil during an Epidemiology meeting using an interactive poster where participants answered about the risk of dying from breast cancer and other causes in the next ten years. ${ }^{7}$ And the same question has been posed in many of our post-graduate classes (PN), and women always overestimate the risk, as did the American women.

Assuming most women in this American study have already heard about the numbers "one in ten," they apparently believed the numbers referred to the next ten years and not to the cumulative lifetime risk at age 85; they have also mistaken incidence for mortality. ${ }^{5}$

Besides this question about the risk of dying in the next ten years, these women answered the following question: "Imagine 1,000 women exactly like you. Among them, how many do you think would die from breast cancer in the next ten years if they received mammography or medical examination screening every year?" (it is worth noting that more than $90 \%$ of them had already had at least one mammography, even though they were under 50 , the age at which mammography starts showing some benefit). Remember that they believed the risk of dying from breast cancer in the next ten years was 100 in 1,000. In this scenario with mammography, they believed 60 would have their lives saved, that is regular screening would save 60 lives for every 1,000 women. First, for women at their age, under 50, there is no evidence that mammography screening saves any lives. Considering the evidence of benefit for women aged 50-70, which is approximately one life saved for every 1,000 screened, then they overestimated the benefit 60 times. These women had definitely been deceived. ${ }^{5}$ To sum up, see Table.

\section{Cognitive biases demonstrated by Daniel Kahneman}

Besides the illusion of certainty (overconfidence and exaggerated conviction), there are other innate distortions inherent to human beings that hinder risk communication and interpretation. Daniel Kahneman suggested there are two thinking systems, system 1 or fast thinking, and system 2 or slow thinking. So, our brain has faster and slower answers, and these two basic ways of reaction affect the way we deal with mathematical questions. System 1, fast, is more intuitive, and system 2, slow, is more reflexive. ${ }^{6}$ It would be a waste of time to use system 2 all the time. More than that, it would be dangerous to use system 2 when speed is crucial. Also, the law of the least effort is adaptive. So, system 2 can be lazy. Thus, we tend to use system 1, fast and intuitive, automatically. This makes sense since system 1 is safer for decisions that should be taken immediately and under uncertainty. However, system 1 sometimes leads us to wrong interpretations. ${ }^{6}$

Table. Risk of dying from breast cancer in the next ten years in a group of well-educated American women, aged 40-49, with no history of breast cancer.

\begin{tabular}{lc}
\hline Perspectives & Risk \\
\hline Risk of dying & 100 in 1,000 \\
What women believe & 5 in 1,000 \\
What the evidence says & \\
Lives saved due to mammography screening & 60 in 1,000 \\
What women believe & 1 in 1,000 \\
What the evidence says*
\end{tabular}

*Evidence of benefit is based on women aged 50-70. There is no evidence that screening saves lives of women aged 40-49. Data based on Gigerenzer. ${ }^{5}$ 
Our emotions are particularly useful for our survival and success in life and act as clues that help us in decision-making and behaviour choice. They alert us about the relevant aspects of our environment that may either threaten us or represent opportunities for us to prosper. Usually, positive emotions encourage us, whereas negative ones inhibit us. The framing bias occurs when positive or negative statements of the same reality evoke different interpretations and behaviours. People, in general, are victims of framing bias, including physicians. For example, the decision by physicians to perform or not a dangerous surgical procedure appeared to differ depending on whether the information they received about the risk of the procedure was framed in a positive or negative format, for instance, 90\% survival versus $10 \%$ mortality risk. ${ }^{6}$

One way to make people believe in falsehoods is frequent repetition because familiarity is not easily distinguished from truth. When people have to decide on a specific situation that the outcome is uncertain, and they do not have or do not remember the information about the likely outcomes in such a situation, they tend to follow any recommendation that is often repeated and thus familiar to them. In these situations, they follow the sense of cognitive ease typical of familiarity. ${ }^{6}$

The anchoring effect is quite common in all areas. It occurs when people consider a particular value for an unknown quantity before estimating that quantity. The result is that the estimates are close to the number that people considered. One example is when half of the participants were asked if when Gandhi died he was more than 114 years old, and the other half if "when Gandhi died he was more than 35 years old. As most participants did not know the age at which Gandhi died, the former gave a much higher estimate of his age at death than the latter. The values of 114 and 35 acted as anchors to the estimated values. The anchoring effect has been demonstrated in numerous ways. Even experienced judges were victims of an anchoring effect of $50 \%$ in an experiment in which a pair of biased dice influenced their decision about the length of a prison sentence they would give to a woman who had been caught shoplifting. ${ }^{8}$

\section{Cognitive biases are automatic and unconscious, so how is it possible to mitigate their undesirable effects on risk interpretation in the context of clinical practice?}

Most clinical decisions are taken under uncertainty. Any health professional treats individual patients and takes clinical decisions individually, but they cannot predict the outcome in one individual patient. They can only know the probability that similar patients with similar problems will recover or not. This uncertain world invites human beings to make decisions based on heuristics, which gives rise to the cognitive biases explained above.

To improve risk interpretation and communication, health professionals must be aware of cognitive biases. This is a matter of attitude. A more attentive, effortful, prudent, less intuitive, and illusory attitude, which is innate to human beings, is a prerequisite for a more rational clinical practice.

More importantly, some forms of risk communication enhance understanding; others do not. Some forms, we suggest, reinforce cognitive bias, while others weaken them. In the words of Gigerenzer, some forms of communicating risks lead to clouded thinking while others facilitate clear thinking. ${ }^{5}$ The remainder of this article will present these forms of risk communication.

Innumeracy - a consequence of an evolutionary mismatch

Maybe the most difficult obstacle to overcome, after the illusion of certainty and the cognitive biases, is innumeracy or difficulty to think with numbers. This difficulty probably arises from a mismatch of ancestral adaptations of the brain having to deal with modern environments, which are quite different from the ancestral ones. When human beings' adaptations to deal with numbers were selected, the environment demanded the ability to deal with small numbers and natural frequencies. Thus, big numbers, percentages, and conditional probabilities are not among the number representation formats naturally selected by the human brain, as these representations were not present in our ancestral environment. 
One analogy is language adaptation. In ancestral environments, there was no writing, which is a very recent invention in human history. Language adaptations, therefore, have not been selected to read and write, but only to speak. That is why it is easy to learn to speak, but difficult to learn to read and write; children do not go to school to learn to speak but to learn to read and write.

Probably, the best treatment for innumeracy lies in restoring the external representation of uncertainties, physicians of risks, to a form that the human mind is adapted to. ${ }^{5}$

\section{Conditional probabilities and relative risk reduction or efficacy are bad forms of risk communication}

There are two quite common, but bad, forms of risk communication: the conditional probability, used to express the accuracy of diagnostic tests, and the relative risk reduction or efficacy, used to express the benefit of interventions. The conditional probability is the risk that the disease is present, given a positive result in the test. The relative risk reduction is the ratio of the risk of getting the disease in the treatment group to the risk in the control group. People, including physicians and dentists, are confused with this kind of information. The confusion caused by conditional probabilities and relative risk reduction can be mitigated by replacing them with natural frequencies. ${ }^{5}$ Let us look at the numbers.

Physicians and dentists misinterpret the results of diagnostic tests and efficacy of treatments

Forty-eight physicians took part in an experiment - including radiologists, gynaecologists, internists, dermatologists, among others, with an average of 14 years of professional experience, from two German cities and working in hospitals or private practice. ${ }^{9}$ They were asked to estimate the chances of breast cancer in a woman aged 40 to 50 years old, given a positive mammogram in a routine screening. Half of them received the information in conditional probabilities and the other half in natural frequencies. ${ }^{9}$ Only two of the 24 physicians in the conditional probabilities group gave the correct answer, in comparison to 11 of the 24 physicians in the natural frequencies group.
Eight and 19, respectively, gave the correct or nearly correct answers, and 16 and 5, respectively, grossly overestimated the number of women with breast cancer. ${ }^{5}$ To sum up, out of the 24 physicians in the natural frequencies group, only 5 were far from the correct answer. In the conditional probabilities group, 16 were far from the correct answers. There were more inconsistencies in the answers and overestimation of breast cancer cases in the latter group.

This finding is not meant to blame physicians or patients for their inability to reason with percentages. The lesson to be learnt is that we should represent the risks in textbooks, articles and clinical practice in a way that comes naturally to the human mind. The use of natural frequencies is a simple and effective way of improving physicians' diagnostic insight. ${ }^{5}$

\section{Do dentists have the same difficulty as Physicians?}

An experimental study was carried out with 101 dentists from different specialties, undergoing a Master's or Ph.D. degree, with a mean age of 29 years and 5 years of professional experience. ${ }^{10}$ Fifty-two dentists received the information of risk in conditional probabilities, and 49 received the same information in natural frequencies. They were asked to estimate the chances of a cavity in an adult given a positive bitewing radiograph, that is radiolucency indicating the presence of interproximal caries requiring a restoration. The following information refers to asymptomatic adults. The statement read by the dentists in the conditional probabilities' group was:

\footnotetext{
"The probability that one of these adults had interproximal caries requiring restorative treatment, confirmed by tooth separation and direct visual and tactile examination, was $8 \%$. The probability of having a positive bitewing radiograph among adults who have a confirmed interproximal caries is $90 \%$. The probability of having a positive bitewing radiograph among adults without interproximal caries is $8 \%$. Imagine an adult who had a positive bitewing radiograph in the survey. What is the probability that he actually has interproximal caries?"
}

The statement read by the dentists in the natural frequencies' group was: 
"Eight in 100 of these adults had interproximal caries requiring restorative treatment, confirmed by tooth separation and direct visual and tactile examination. Among these 8 adults with confirmed interproximal caries, 7 will have a positive bitewing radiograph. Among the 92 adults without interproximal caries, 7 will have a positive bitewing radiograph. Imagine an adult who had a positive bitewing radiograph in the survey. What is the probability that he actually has interproximal caries?"10

The correct answer was 7 in 14 adults with positive bitewings, in fact, have interproximal caries, which corresponds to 1 in 2, or $50 \%$ (Figure 1). Only one of the 52 dentists in the conditional probabilities' group gave the correct answer, in comparison to 13 of the 49 dentists in the natural frequencies' group. Correct plus nearly correct answers were given by one and 20 dentists in the conditional probabilities and natural frequencies' group, respectively. Forty and 20 dentists in the conditional probabilities and natural frequencies groups, respectively, overestimated the numbers of adults with interproximal caries. The probabilities of caries underestimation were similar, $21 \%$ and $29 \%$, respectively (Figure 2 ). ${ }^{10}$

Similar to what happened to physicians, ${ }^{5}$ these two representations illustrate how easier it was for dentists to reason with natural frequencies rather than with conditional probabilities (Figure 3).
The same dentists that participated in the study about interproximal caries diagnosis with bitewing radiographs took part in a study about the benefits of dental treatment. ${ }^{11}$ They were asked to estimate how many patients with periodontitis would avoid tooth loss if they underwent periodontal treatment. The same hypothetical scenario was presented in two formats. The group of percentages and relative risk reduction received the following statement:

"Suppose that $4 \%$ of adults with periodontitis will suffer multiple
tooth losses due to periodontitis. Compared to no treatment, the
non-surgical periodontal treatment of adults with periodontitis
has an efficacy of $25 \%$ in avoiding multiple tooth losses and,
when associated with taking antibiotics, the efficacy is $50 \%$ ".

The other group received the same information in natural frequencies and absolute risk reduction, that is the format that evokes unclouded thinking. The statement for this group was:

\footnotetext{
"Suppose that for 100 adults with periodontitis 4 will suffer multiple tooth losses due to periodontitis. If exposed to non-surgical periodontal treatment, 3 , instead of 4 , will suffer multiple tooth losses. And, finally, if this treatment includes taking antibiotics, 2, instead of 4 of these patients will suffer multiple tooth losses".11
}

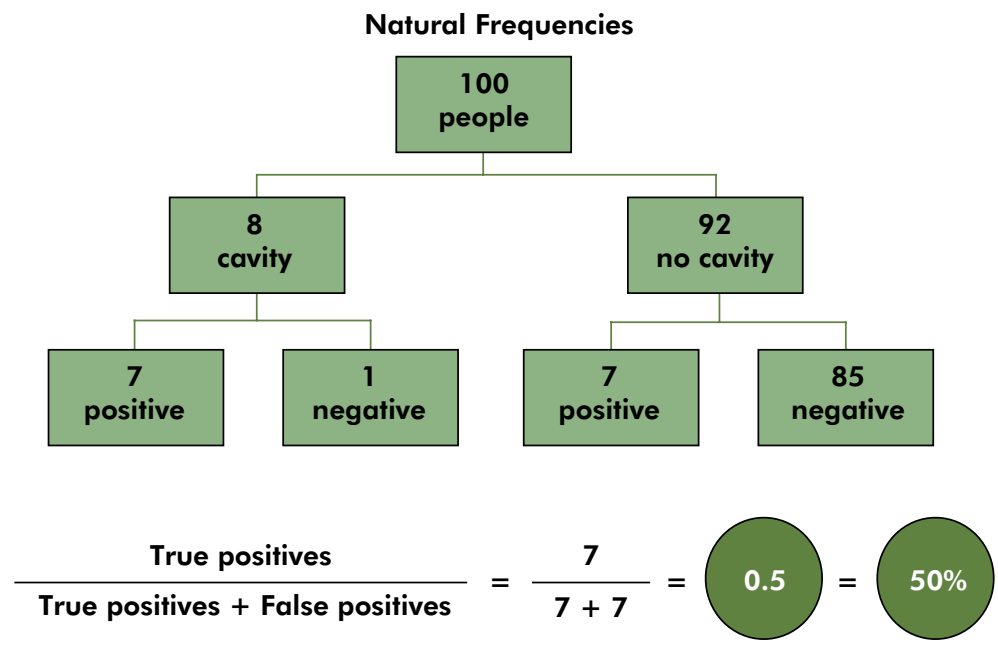

Figure 1. Dentists who received the information in natural frequencies found it easier to estimate the chances of interproximal caries given a positive bitewing radiograph. The reason is that they had to pay attention only to two numbers: the number of adults with a positive bitewing radiograph and caries (7) and the number of adults with a positive bitewing radiograph without caries (7). Adapted from Nadanovsky et al. ${ }^{10}$ with permission. 


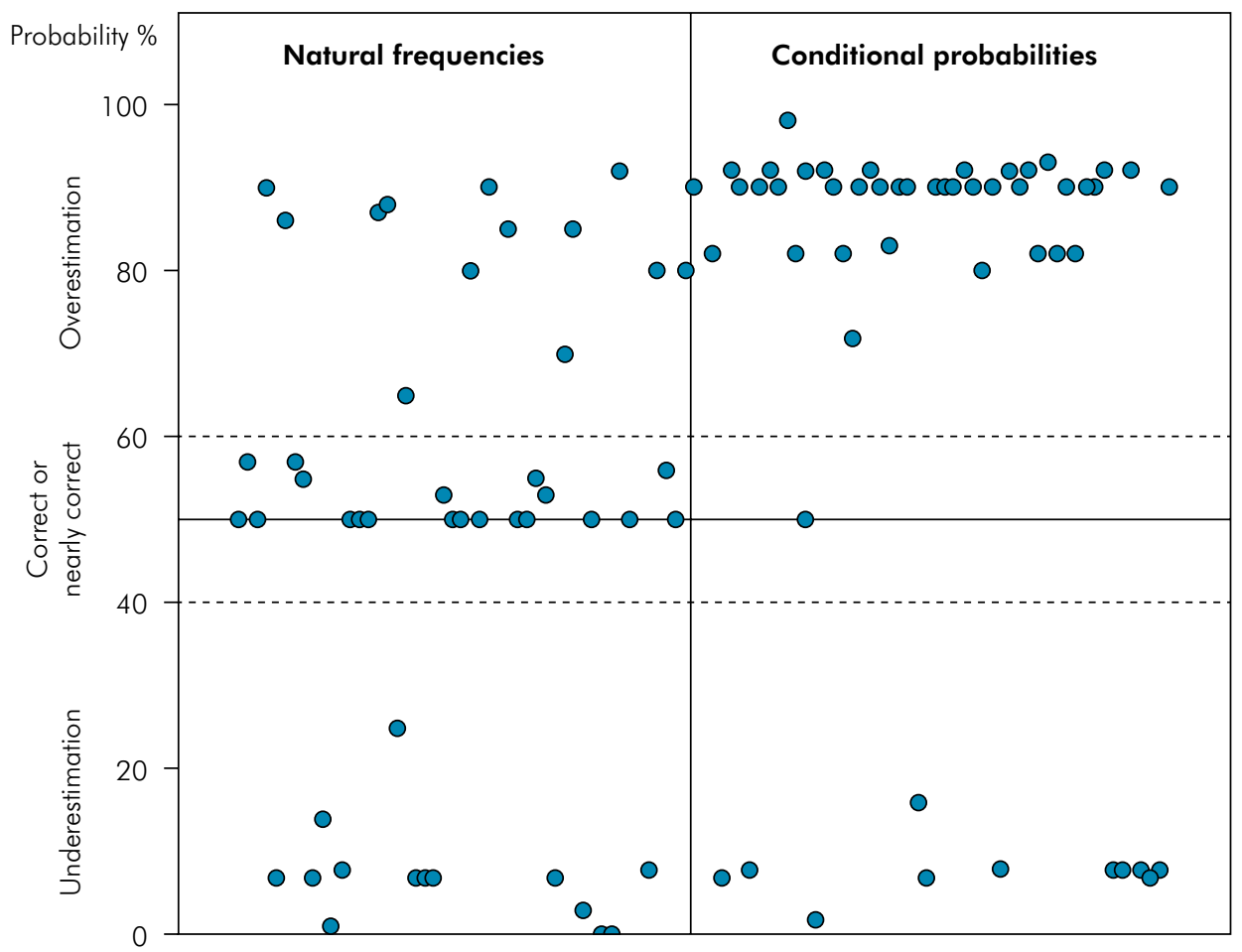

Figura 2. Answers given by all the dentists according to the format in which they received the diagnostic test accuracy information. The correct answer in this hypothetical scenario was $50 \%$, the nearly correct answers $40 \%-49 \%$ or $51 \%-60 \%$, underestimation less than $50 \%$ and overestimation greater than $50 \%$. From Nadanovsky et al. ${ }^{10}$ with permission.

\section{Conditional Probabilities}

\begin{tabular}{c}
$\mathrm{p}($ disease $) \times \mathrm{p}(\mathrm{pos} \mid$ disease $)$ \\
\hline $\mathrm{p}($ disease $) \times \mathrm{p}(\mathrm{pos} \mid$ disease $)+[1-\mathrm{p}($ disease $)] \times \mathrm{p}(\mathrm{pos} \mid$ nodisease $)$ \\
$\left.\frac{0.08 \times 0.9}{0.08 \times 0.9+[1-(0.08)] \times 0.08}=0.495\right)$
\end{tabular}

Figura 3. In the formula of probabilities, the proportion of adults with interproximal caries in the population is $8 \%$, the proportion of positives with interproximal caries is $90 \%$ and the proportion of positives without interproximal caries is $7.6 \% \approx 8 \%$. Adapted from Nadanovsky et al. ${ }^{10}$ with permission.

Both groups had to answer two questions. The first one was: "For each 100 adult patients with periodontitis, how many will avoid multiple tooth losses due to the non-surgical periodontal treatment without antibiotics?" The correct answer is one. If without treatment, four patients lose teeth and with the non-surgical periodontal treatment without antibiotics, three patients lose teeth, then four minus three equals one.
The second question was the following: "For each 100 adult patients with periodontitis, how many will avoid multiple tooth losses due to the non-surgical periodontal treatment associated with taking antibiotics?" The correct answer is two. If without treatment four patients lose teeth and with the non-surgical periodontal treatment associated with taking antibiotics two patients lose teeth, then four minus two equals two. ${ }^{11}$ 
Most dentists did not correctly interpret the benefit of treatment in the hypothetical scenario in this study (Figure 4). But correct interpretations were more frequent in the natural frequencies than in the relative risk reduction group. Between one in three (33\%) and one in two (50\%), dentists interpreted the hypothetical scenario correctly in the natural frequencies group while only one in four (25\%) dentists did it in the relative risk reduction group. ${ }^{11}$

Possibly the most valuable finding of this study came not from the correct answers, but from the incorrect ones. The incorrect answers unveiled that the reason why dentists were confused and misinterpreted the benefit of the treatment was because they did not pay attention to the base rate, that is, the risk of multiple tooth loss in patients with periodontitis in the hypothetical scenario of this study. They very clearly fell prey to the baserate neglect. ${ }^{5,12}$ This neglect was apparent in both the relative risk reduction and the natural frequencies groups. In the relative risk reduction group, many

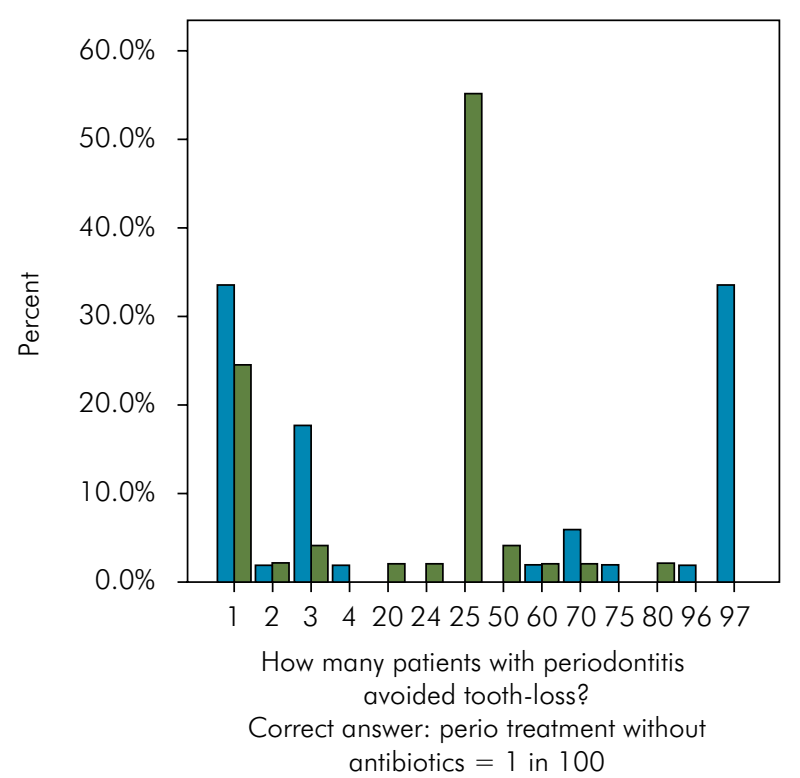

dentists clung to the information that the efficacy of treatment was $25 \%$ and $50 \%$, interpreting that for every 100 patients, 25 and 50 would avoid multiple tooth losses, respectively, in the scenarios without and with antibiotics. They did not realize that those $25 \%$ and $50 \%$ efficacy had to be applied to the 4 in 100 risk of multiple tooth losses so that $25 \%$ of 4 is 1 and $50 \%$ of 4 is 2 .

Similarly, the rationale of many dentists in the natural frequencies group was the following: given that for 100 patients, 3 and 2 suffered multiple tooth losses in the scenarios without and with antibiotics, respectively, this means that the other patients, that is, 97 and 98 , would not suffer multiple tooth losses. Thus, 97 and 98 would benefit from treatment, as all 100 patients underwent treatment. They neglected the fact that most patients, that is, 96, would not suffer multiple tooth losses anyway, even without any treatment. They were under the spell of the illusion of certaint $y^{5}$ - in this case, the illusion that every patient that was treated and did not suffer the

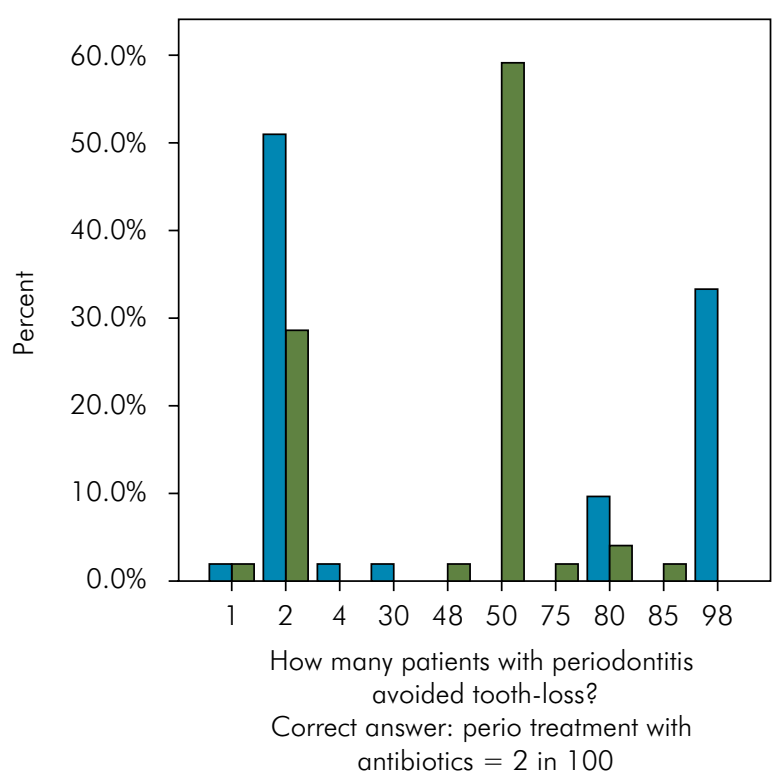

Communication format

Absolute numbers

Percentages

Figure 4. Frequency distribution of the answers given by the dentists to the question of how many patients will avoid multiple tooth losses due to the non-surgical periodontal treatment without $(A)$ or with antibiotics $(B)$. The right answer to non-surgical periodontal treatment without antibiotics is one and with antibiotics is two. Horizontal axis shows the answers given by the dentists. The bars show the percentages of each answer. Blue bars represent dentists in the natural frequencies' group and green bars represent dentists in the percentages' group. 
undesirable outcome benefited from the treatment. However, an important finding was that this bias was more accentuated in the relative risk reduction than in the natural frequencies group, with approximately 20 to 30 fewer dentists (per one hundred) showing signs of the base-rate neglect in the latter than in the former group.

Communicating benefit of treatment using natural frequencies facilitated clear thinking among dentists because, in this format, fewer dentists neglected the base-rate information. Industry, journalists, health professionals, editors, and authors of scientific journals should use natural frequencies and absolute risk reduction instead of the formats in percentages and relative risk reduction.

Future studies should test new ways to try and focus people's minds on the base-rate. Visual aids and qualitative research techniques may be useful avenues to explore to find successful strategies to facilitate risk communication in addition to the natural frequencies format.

\section{The prevented fraction is a common but misleading form of expressing the benefit of interventions}

The prevented fraction $(\mathrm{PF})$ is the preferred method of computing DMFT (decayed missing or filled teeth) and DMFS (decayed missing or filled tooth surfaces) data of clinical trials and meta-analyses that evaluate caries as the outcome, as it presumably has an easy interpretation and allows the combination of different methods of measuring caries, such as DMFT (S), DFT (S), dmft (s), dft (s). It is calculated by subtracting the increment of caries in the test group (It) from the increment of caries in the control group (Ic) and dividing this difference by the increment of caries in the control group, that is it calculates the percentage of the increment of caries in the control group that can be reduced by the intervention/treatment. The formula is simple: $\mathrm{PF}=$ (Ic - It) / Ic.

However, as it is a relative measure, the PF fails to show the true differences in the caries increment observed between the groups, and an apparently substantial PF may be clinically irrelevant in terms of actual caries reductions. Moreover, PF and its confidence intervals' calculations are very unstable when studies show caries increment close to zero. For example, in a recent meta-analysis about the effectiveness of fluoride varnish on caries prevention in preschoolers, ${ }^{13}$ one clinical trial showed a mean increment of caries of $0.42 \mathrm{dmft}$ in the control group and $0.30 \mathrm{dmft}$ in the test group. The PF, in this case, was $29 \%$, meaning that there was $29 \%$ of caries reduction at tooth level attributed to the fluoride varnish. Still, the difference in the mean increments was modest, only 0.12 tooth, that is a difference that is less than one-fifth of a tooth. Also, had we used one decimal case instead of two, the PF would be 25 and not $29 \%$. Thus, $\mathrm{PF}$ is a misleading way of communicating risk or risk reduction and should be avoided. Instead, researchers should use natural frequencies formats and absolute numbers to communicate the benefits of interventions in studies of dental caries.

\section{Bad risk communication also leads to the adoption of relatively ineffective preventive strategies}

It is naturally difficult for people to understand the relationship between population and individual risk. Hence, good risk communication is of utmost importance to promote clear thinking in this context, that is when population and individual risks are evaluated to implement preventive health programmes. There are preventive strategies that are greatly beneficial for the population but at the same time of little benefit for the individual. To explain this counterintuitive phenomenon, Geoffrey Rose coined the term "the prevention paradox". ${ }^{14}$ For example, seat belts are highly beneficial for the population as they substantially reduce the total number of deaths and serious sequela due to car crashes. However, the probability of an individual to benefit from seat belts is small - to benefit, not only does he have to suffer a car crash but also this car crash cannot be too weak (in this case seat belts are unnecessary) nor too strong (in which case seat belts are insufficient). An event of this nature has a low probability of occurring in an individual's life, but a great probability of occurring in a population. Thus, we need a special cognitive effort when communicating data of these two types of risks, the individual and the population, to avoid common 
mistakes and misinterpretations that often lead to relatively ineffective preventive strategies. ${ }^{14}$

It is common to say that, due to the decline of caries, most caries lesions are concentrated in a minority of children, the so-called high-risk children. This polarization has been named the 80:20 phenomenon, in which supposedly $80 \%$ of caries lesions would be found in $20 \%$ of children. In at least one occasion, this phenomenon has been investigated and considered exaggerated. ${ }^{15}$

Using data from the American "National Preventive Dentistry Demonstration Programme (NPDDP)," Batchelor and Sheiham investigated the distribution of new caries lesions in seven-year-old children during four years of follow-up. In a subsample of high-risk children with a baseline DMFS of five or more and not exposed to fluoridated water and dental sealants, there was an increment of more than four new decayed surfaces; among those of low risk (no caries at baseline), the increment was approximately two new decayed surfaces. However, more than $64 \%$ of all new caries lesions occurred in children with no caries at baseline, whereas less than one percent of all new caries lesions occurred in high-risk children, that is those with a DMFS of five or more at baseline. ${ }^{16}$

The communication problem here is that high-risk children do develop, on average, more caries lesions than the rest of the children. That is why it is said that they concentrate most caries lesions. However, as these children are a minority in the population, larger amounts of caries lesions accumulate in lowrisk children, who are the majority.

This confusing communication encourages the adoption of preventive strategies that seek to identify high-risk children and aim the preventive measures at them. At the same time, it discourages population strategies that encompass all children, including the low-risk ones, who develop most new caries lesions in the population. Therefore, the preventive strategies adopted are those with less potential to reduce the incidence of caries in the population.

\section{Conclusion}

We have seen that bad risk communication raises problems in the interpretation of diagnostic tests and treatment benefits. Also, it creates difficulty for the understanding of individual and population risks, which leads to the adoption of relatively ineffective preventive strategies. The implications of bad risk communication for medical and dental decisionmaking are disastrous. To try and solve this problem, it is necessary to recognise its existence.

Health professionals should overcome the illusion of certainty, that is the exaggerated but delusional convictions about the certainty of diagnostic tests accuracy and treatment benefits. Also, we should remain alert regarding our innate cognitive biases (that often results from our natural heuristics) that may be useful in many contexts, but at the same time may lead us to take disastrous clinical decisions. This is a matter of mental attitude, which includes striving against the comfort of following our intuition instead of making an anti-natural effort to think slowly and reflexively about the numbers.

Besides this mental attitude, and to reinforce it, we should change the way we communicate risks by turning the numbers into formats that the human brain finds it easy to understand. The main methods so far discovered to facilitate a clearer risk communication in the context of clinical research are to emphasize the base rates of the events and to use absolute numbers, that is to use natural frequencies, instead of percentages and conditional probabilities.

\section{References}

1. Ilic D, Neuberger MM, Djulbegovic M, Dahm P. Screening for prostate cancer. Cochrane Database Syst Rev. 2013 Jan;(1):CD004720. https://doi.org/10.1002/14651858.CD004720.pub3

2. Kale MS, Korenstein D. Overdiagnosis in primary care: framing the problem and finding solutions. BMJ. 2018 Aug;362:k2820. https://doi.org/10.1136/bmi.k2820 
Risk communication in the context of clinical research

3. Drazen's dozen: articles that changed practice since 2000. N Engl J Med. 2019 May.

4. Zauber AG, Winawer SJ, O'Brien MJ, Lansdorp-Vogelaar I, Ballegooijen M, Hankey BF, et al. Colonoscopic polypectomy and long-term prevention of colorectal-cancer deaths. N Engl J Med. 2012 Feb;366(8):687-96. https://doi.org/10.1056/NEJMoa1100370

5. Gigerenzer G. Reckoning with risk: Learning to live with uncertainty. London: Penguin; 2002.

6. Kahneman D. Thinking, fast and slow. New York: Farrar, Straus aand Giroux; 2011.

7. Cunha-Cruz J, Nadanovsky P. Comunicação do risco: qual é o seu risco de morrer nos próximos 10 anos? In: VI Congresso Brasileiro de Epidemiologia, 2004, Recife, PE. Rev Bras Epidemiol. 2004;7(supl 1):1016.

8. Englich B, Mussweiler T, Strack F. Playing dice with criminal sentences: the influence of irrelevant anchors on experts' judicial decision making. Pers Soc Psychol Bull. 2006 Feb;32(2):188-200. https://doi.org/10.1177/0146167205282152

9. Hoffrage U, Gigerenzer G. Using natural frequencies to improve diagnostic inferences. Acad Med. 1998 May;73(5):538-40. https://doi.org/10.1097/00001888-199805000-00024

10. Nadanovsky P, Santos AP, Lira-Junior R, Oliveira BH. Clinical accuracy data presented as natural frequencies improve dentists' caries diagnostic inference: evidence from a randomized controlled trial. J Am Dent Assoc. 2018 Jan;149(1):18-24. https://doi.org/10.1016/i.adaj.2017.08.006

11. Nadanovsky P, Santos AP, Lira-Junior R, Oliveira BH. Dentists' inferences about treatment efficacy using absolute numbers and percentages. 2018 AADR/CADR 47th Annual Meeting Fort Lauderdale, Fla., USA. J Dent Res. 2018;97(Spec Iss A):1483.

12. Cosmides $L$, Tooby J. Are humans good intuitive statisticians after all? Rethinking some conclusions from the literature on judgement under uncertainty. Cognition. 1996;58(1):1-73. https://doi.org/10.1016/0010-0277(95)00664-8

13. Sousa FS, Santos AP, Nadanovsky P, Hujoel P, Cunha-Cruz J, Oliveira BH. Fluoride varnish and dental caries in preschoolers: A systematic review and meta-analysis. Caries Res. 2019;53(5):502-13. https://doi.org/10.1159/000499639

14. Rose G. Sick individuals and sick populations. Int J Epidemiol. 1985 Mar;14(1):32-8. https://doi.org/10.1093/ije/14.1.32

15. Tickle M. The 80:20 phenomenon: help or hindrance to planning caries prevention programmes? Community Dent Health. 2002 Mar;19(1):39-42.

16. Batchelor PA, Sheiham A. The distribution of burden of dental caries in schoolchildren: a critique of the high-risk caries prevention strategy for populations. BMC Oral Health. 2006 Jan;6(1):3. https://doi.org/10.1186/1472-6831-6-3 